\title{
Ressources ultimes d'eau potable en cas de crise de pollution majeure : rôle des gisements d'eau embouteillée
}

\author{
J.J. Collin, J.P. Comte, J.R. Daum, M. Lopoukhine \\ BRGM - Centres thématiques Eau et Eaux thermo-minérales
}

\author{
M. Mesny
}

Haut fonctionnaire de Défense, Ministère de l'Environnement

\section{I $\square$ RAPPEL DE QUELQUES CHIFFRES}

La consommation d'eau potable en France est en moyenne de 250 litres par jour et par habitant, soit 14 millions de $\mathrm{m}^{3}$ par jour et plus de 5 milliards de $\mathrm{m}^{3} / \mathrm{an}$. Les besoins en boissons ne dépassent pas 2 litres par jour et par personne, couverts en moyenne de la façon suivante : eau embouteillée $(0,25)$, lait $(0,20)$, vin, bière, jus de fruit et divers $(0,40)$, eau potable au robinet $(1,15)$.

Les besoins de survie (boissons + cuisine + hygiène élémentaire) sont estimés à 4 litres d'eau potable par jour et par habitant.

\section{LES EAUX SOUTERRAINES PROTÉ- GÉES}

La sécurité de la distribution de l'eau potable peut être mise en péril par divers facteurs. La pollution accidentelle, les désordres géotechniques affectant des canalisations, voire des actes de malveillance, ont en commun leur caractère ponctuel et peu durable ou « réparable ", et sont donc à traiter au cas par cas et de manière adaptée au contexte physiographique et socio-économique local.

Actuellement, plus des trois quarts de l'eau distribuée pour l'alimentation en eau potable provient soit des eaux de surface, soit de nappes superficielles, alluviales ou phréatiques : en tout état de cause, il s'agit de ressources particulièrement vulnérables. L'enjeu est donc important. Certes, la réglementation prévoit la protection de ces captages contre les effets de pollutions accidentelles ou diffuses et pratiquement de caractère relativement local. Il en est tout autrement des effets de grandes catastrophes ou des conséquences de conflits armés qui seraient susceptibles de rendre impropres à la consommation, voire dangereuses, la plupart de ces ressources.

Bien que le risque militaire (nucléaire, bactériologique et chimique) ait évolué ces dernières années avec le contexte géopolitique, le risque accidentel nucléaire, lui, demeure très présent, comme l'a démontré l'accident de

Ultimate resources of drinking water in the event of a major pollution crisis : the role of bottled water

In the event of a serious and widespread pollution incident - on the level of the "Chernobyl cloud" most of the drinking water resources in France could be contaminated : surface water immediately, ground water in a few days... or a few months.

Therefore on the initiative of the Ministry of the Environment's Director for Defence, a study has been initiated as to what might be qualified as "final emergency resources ". An inventory and map of protected resources have been prepared. In this context it seems reasonable to show bottled water as a resource meeting the necessary protection criteria. However it seems that these criteria are not all, nor always, relevant for defining $a$ " ultimate emergency resource 》 not contaminated by a major incident.

This article outlines a typology of situations and defines the main criteria necessary for bottled water to be able to constitute an ultimate resource. 
Tchernobyl dont les conséquences, dramatiques en Ukraine, ont inquiété certains de nos voisins. Le niveau d'alerte a été frôlé dans le Sud-Est de notre pays. Dans la suite de l'article, nous appellerons donc risques « N.B.C " les menaces de pollution catastrophique généralisée.

L'hypothèse d'un nouvel accident, dans les pays d'Europe de l'Est, n'est pas à exclure, compte tenu de l'état de leur parc de centrales nucléaires. Malgré la série de dispositifs de sécurité en vigueur dans les pays occidentaux, on ne peut pas non plus y écarter totalement l'idée d'un accident majeur.

L'eau "grande dénonciatrice des secrets de la Terre » serait à la fois la victime et le vecteur principal d'une telle pollution, véhiculée d'abord sous forme d'aérosols. Ruissellement et infiltration des pluvio-lessivats, voire l'enchaînement de ces deux phénomènes, pourraient contaminer :

- immédiatement les eaux de surface libres,

- avec un léger différé, les nappes phréatiques ou nappes libres proches de la surface,

- avec un différé de quelques mois ou plus, les nappes libres «profondes ".

Le transfert par aérosol aurait pour conséquence une large distribution spatiale (selon le régime météorologique du moment). Ensuite, la fixation sur les très grandes surfaces foliaires des végétaux, puis le lessivage de ces surfaces et des sols, et enfin l'infiltration dans le sous-sol contribueraient alors à une pollution de tous les aquifères phréatiques de vastes portions du pays. Le retard à l'infiltration, en fonction de l'épaisseur de la zone non saturée, peut apporter, en certaines zones, un facteur atténuant.. mais ne fait que temporiser le péril.

Dans le cadre de l'étude de scénarios de crises diverses - dont font partie les risques N.B.C. évoqués ci-dessus le Haut Fonctionnaire de Défense auprès du Ministère de l'Environnement a chargé le BRGM (Service Public) d'identifier les ressources qui seraient susceptibles d'offrir à la population une eau potable de secours. Dans une première phase, la réflexion a porté sur les ressources protégées ou "d'ultime recours », dans une perspective essentiellement d'inventaire.

Cette démarche, fondée sur une méthode cartographique, a largement fait appel à des données sur les nappes profondes dont l'existence est attestée par diverses études géologiques, mais qui sont peu exploitées et à ce titre mal connues. La nature même du problème à résoudre (situation de crise temporaire) permet d'envisager le recours à des ressources qui n'offrent pas toutes les qualités exigées des nappes exploitées en régime normal. On pourra imaginer l'usage de ressources épuisables à moyen terme, de productivité non économique, de qualité chimique imparfaite, voire même de ressources qui seraient polluables à l'issue d'un délai de quelques mois après le début de la crise et de la contamination des eaux de surface.

L'hydrogéologie des ressources d'ultime recours peut donc être qualifiée «d'hydrogéologie revisitée » ou d'hydrogéologie «à rebours". A côté de ces nappes méconnues, des gisements très classiques et déjà très sollicités peuvent satisfaire la demande spécifique.

L'extrême diversité des ressources en eau souterraine de France a permis de dresser une carte de France au $1 / 1500000$ des ressources en eau (souterraine) protégées ; la figure $I$ en présente un extrait. Certaines régions apparaissent favorisées, avec, notamment, les grandes nappes captives d'Aquitaine, du Bassin de Paris et de Lorraine, d'autres sont quasi dépourvues de solutions de secours (Bretagne et Massif Central), d'autres enfin présentent des ressources diverses très variables en extension et en potentialités. Certaines doivent leur protection à une réelle couverture étanche et une alimentation latérale éloignée, d'autres - sous réserve de vérification par des techniques isotopiques ou de traçage - bénéficient d'une recharge différée par un long transit dans une zone non saturée épaisse ou dans un aquifère poreux, perméable mais anisotrope.

\section{LES EAUX EMBOUTEILLÉES : UNE IMAGE DE PURETÉ}

Parmi les hypothèses envisageables - et notamment retenues par nos voisins suisses - le recours à des stocks d'eau embouteillée peut être une solution pour les tout premiers jours de crise... Cela conduit donc naturellement à imaginer que les eaux «minérales » pourraient avoir, dans ces circonstances, un rôle privilégié, non seulement en phase initiale, par suite de leur conditionnement, mais également en deuxième phase, par suite des caractéristiques propres aux gisements et du haut niveau de qualité hygiénique des eaux commercialisées.

Les eaux embouteillées (et notamment les eaux minérales et de source) doivent en effet satisfaire à plusieurs contraintes techniques imposées par la réglementation, et notamment le décret du 6 juin 1989 :

" une eau minérale naturelle (...) se distingue par sa pureté originelle. (Elle) a été tenue à l'abri de tout risque de pollution (...). Elle témoigne (...) d'une stabilité (...) de ses caractéristiques essentielles (composition, température...) " (article 2).

" une eau de source est une eau d'origine souterraine (...) protégée contre les risques de pollution... " (article $13)$.

Autrement dit, les eaux doivent présenter, quelles que soient les circonstances, une qualité chimique et bactériologique stable, et les eaux de source doivent rester naturellement dans la plage des normes françaises de potabilité.

On peut donc a priori imaginer que les sites d'embouteillage soient considérés comme des lieux de captage de ressources d'ultime recours.

En outre, la production actuelle des eaux destinées à l'embouteillage atteint 4,5 millions de $\mathrm{m}^{3}$ par an (soit 80 litres par habitant), ce qui est très intéressant face à une demande très restreinte d'eau de qualité potable au sens strict ( 5 à 10 litres par jour et par habitant).

Ce qui semble une évidence (la parfaite protection sanitaire des eaux embouteillées) n'est cependant pas une certitude dans toutes les configurations de gisement ni face à tous les types de pollution.

Les critères de qualité "environnementale " de nombreuses sources commercialisées sont fondés, entre autres, sur des conditions de sites naturels traditionnellement «purs".

Le concept de protection est en effet inspiré de celui de la protection des eaux potables banales (AEP)... c'est-àdire que des couches non imperméables mais suffisamment filtrantes assurent, en régime normal, la protection bactériologique et que la qualité des milieux naturels fait le 


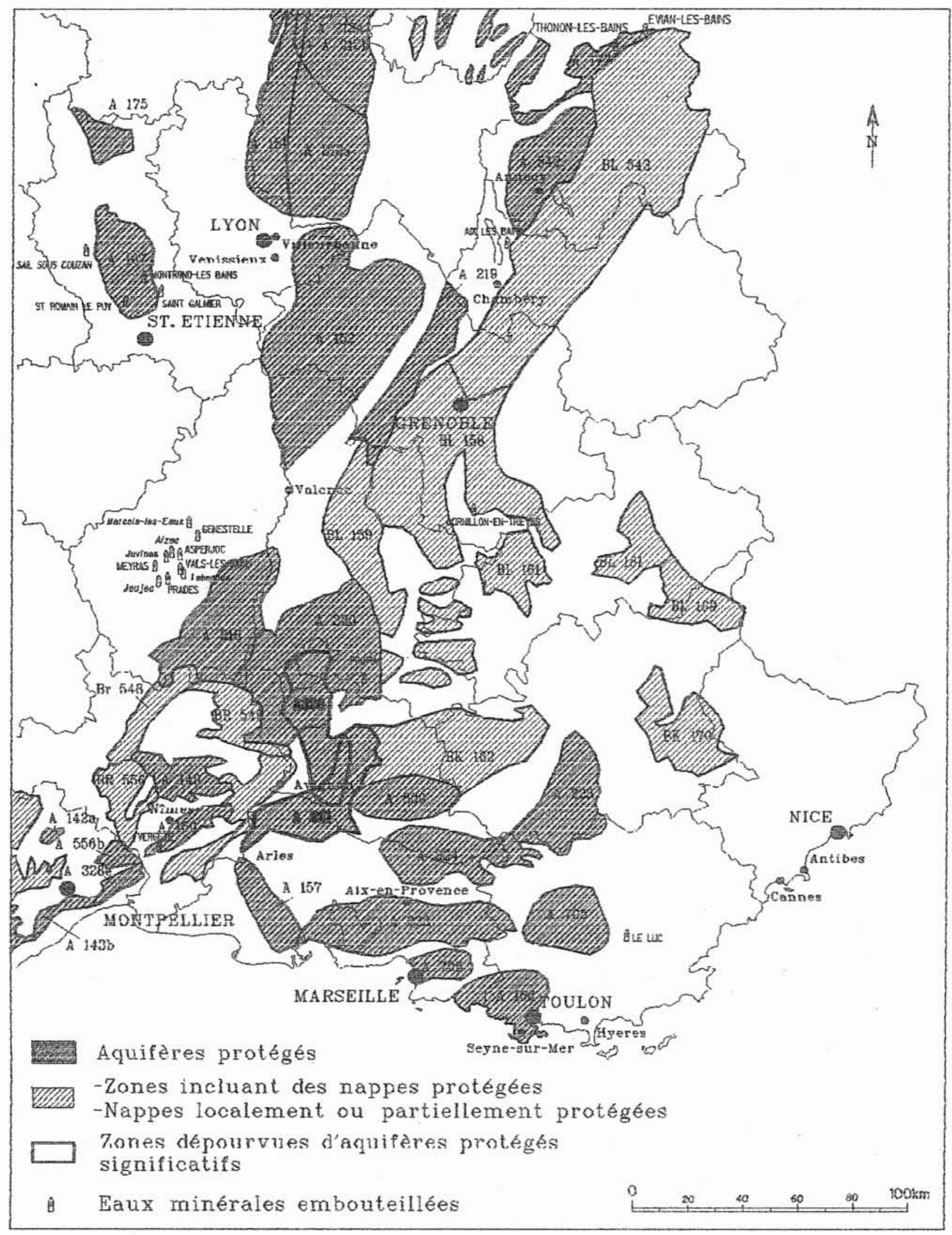

1. Extrait de la carte de France des aquifères protégés. 
reste : des forêts, des alpages, etc. apportent une qualité naturelle, une absence de nitrates ou de pesticides... Le maintien de cette qualité (par le rachat des exploitations agricoles polluantes, par des mesures spéciales d'interdiction, par la prévention d'installations polluantes), fait l'objet de la politique des sociétés d'eau embouteillée... et, à ce titre, les résultats obtenus par celles-ci sont tout à fait capables d'assurer une qualité durable des produits, qui satisfait ainsi aux normes sanitaires et aux lois du marché.

\section{IV — LES EAUX EMBOUTEILLÉES : DES GI- SEMENTS N'ÉCHAPPANT PAS À TOUS LES RISQUES}

Les critères évoqués ci-dessus peuvent s'avérer parfaitement pertinents en régime courant : ils seraient par contre incorrects, voire dangereux, pour une pollution apportée par voie météorique, suivie d'infiltration.

En effet, il en va tout autrement à l'égard de pollutions généralisées qui, venues avec la recharge pluviale, ne pourraient être évitées sur la totalité de l'impluvium, ni être éliminées par les processus qui protègent habituellement les ressources.

De plus, pour nombre d'entre elles, il est fait état d' « âge " relativement important, de l'ordre de quelques années à plusieurs centaines, voire plusieurs milliers d'années ce qui présente apparemment une garantie de noninfiltration récente et, partant, de non-infiltration potentielle d'une pollution éventuelle généralisée.

Cependant, à l'examen. les faits s'avèrent plus compliqués, et il apparaît difficile d'atteindre, pour la plupart des eaux embouteillées, le niveau de certitude a priori requis pour des ressources «d'ultime recours». En effet, plusieurs causes d'incertitudes subsistent même pour les sources les plus connues et les plus distribuées.

\subsection{Cas des mélanges naturels}

La réglementation, actuelle et passée, qui porte sur les sources, et non sur les gisements, a permis l'attribution du label eau minérale à nombre de sources (c'est particulièrement flagrant pour les eaux gazeuses) qui sont en réalité constituées par des mélanges, se produisant naturellement dans le sous-sol, entre plusieurs ressources en eau de caractéristiques parfois bien différentes (le mélange d'un concentré minéralisé et gazeux d'origine profonde, dilué par des eaux de nappes moins profondes est le cas le plus typique).

Dans de tels cas, dont l'identification formelle est relativement récente, le niveau de connaissance acquis reste insuffisant pour pouvoir affirmer avec certitude que chacun des composants, en particulier les eaux issues des formations de surface, peut satisfaire aux critères imposés pour des ressources ultimes.

\subsection{Problème des circulations préférentielles}

Si dans certains cas on admet, suite à des campagnes de mesures isotopiques, que l'âge d'une eau est d'un ordre de grandeur satisfaisant (ex. EVIAN 15 à 18 ans), peut-on affirmer avec certitude que ce chiffre n'est pas une moyenne de valeurs, reflet du mélange d'eaux identiques mais ayant suivi des trajets de durées très différentes. On en a un exemple sur VOLVIC, où suivant les points de prélèvement, on obtient des valeurs variant de 4 mois à plusieurs années, et ce pour une qualité d'eau homogène dans sa composition chimique..

\subsection{Type de captage}

Indépendamment de la qualité de l'eau de recharge on peut également mettre en avant un autre facteur de discrimination, la nature de l'ouvrage de captage.

A priori, seules des eaux minérales captées par des forages tels qu'on les réalise depuis environ une quinzaine d'années devraient pouvoir satisfaire aux critères d'étanchéité et de protection, requis pour la préservation de la ressource en cas de pollution majeure... ce qui élimine du choix les captages de sources, toujours vulnérables.

\subsection{Conception de la chaîne de transport et de condi-} tionnement

La progression très rapide des contraintes d'hygiène imposées par le marché a obligé nombre d'exploitants à moderniser leurs installations pour atteindre des niveaux d'hygiène et de qualité de process exemplaires. C'est par exemple le cas indiscutable de la plupart des grandes marques.

Par contre, ne correspondraient pas au cahier des charges requis :

- d'une part, toutes les lignes de conditionnement en verre (bouteilles fabriquées sur place ou transportées depuis le lieu de production),

- d'autre part, beaucoup de moyennes et la plupart des petites entreprises dont la démarche qualité à l'intérieur de l'usine n'atteindrait pas encore le niveau souhaité pour une ressource ultime.

Cela ne s'applique pas à l'usage des forages comme des ouvrages « banaux ", avec réalimentation d'un réseau.

\section{V— LES GISEMENTS D'EAUX EMBOU- TEILLÉES ET LEUR PROTECTION}

La figure 2 présente la typologie des cas les plus fréquemment représentés dans les gisements d'eau embouteillée. On y trouve :

- des nappes libres apparemment protégées par l'environnement favorable mais non réellement à l'abri d'un risque N.B.C. :

- des nappes libres protégées par la durée du temps de transit vertical de la recharge... et donc protégées du risque N.B.C. immédiat... mais pas de risques plus durables ;

- des nappes libres protégées par une couverture imperméable :

- des nappes captives à alimentation lointaine (qui sont souvent aussi bien exploitées pour l'eau potable que pour l'embouteillage), offrant une bonne protection dans la majorité des cas : 


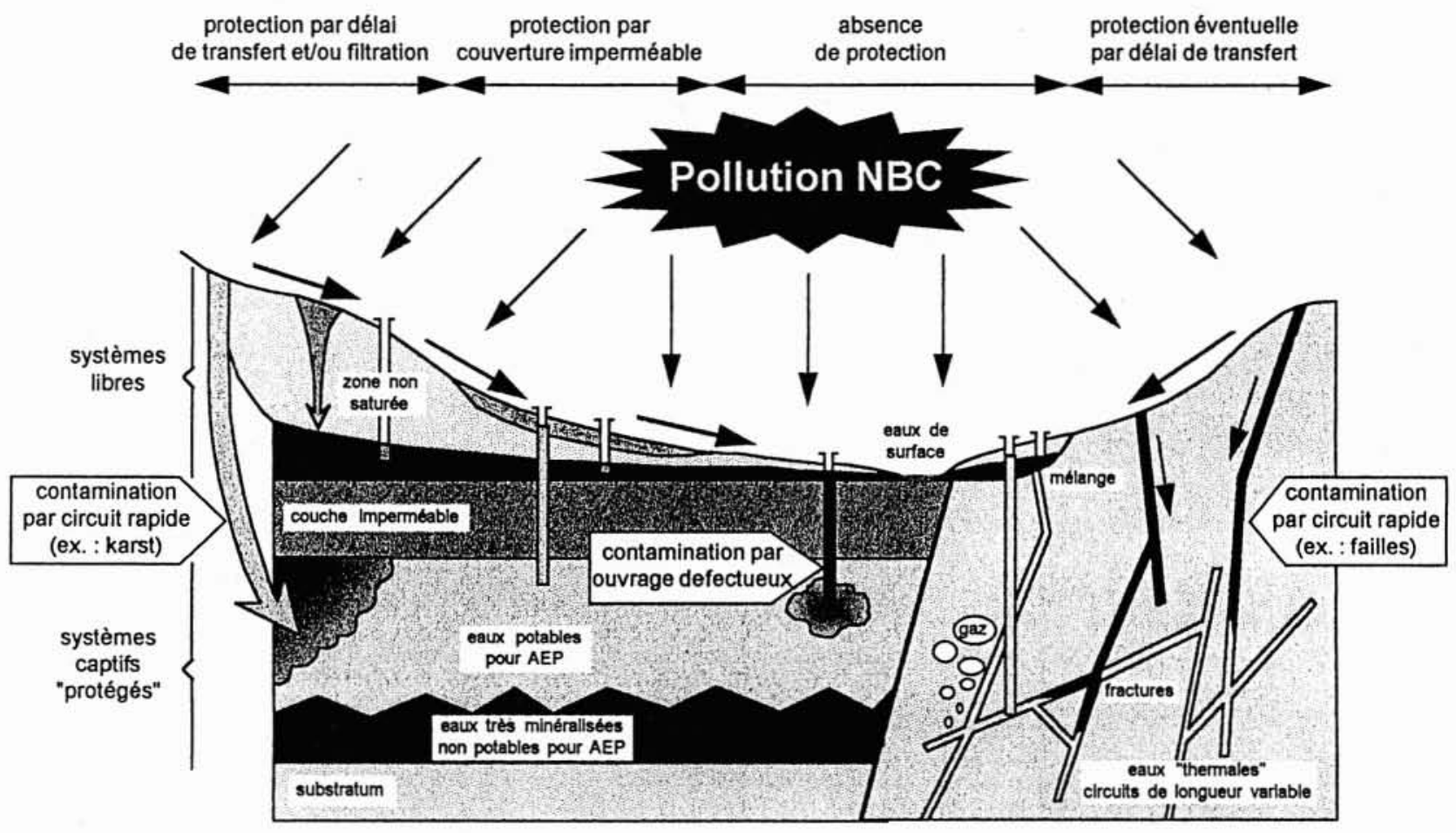

2. Schéma conceptuel de la vulnérabilité et de la protection des aquifères vis-à-vis des pollutions accidentelles météoriques généralisées « N.B.C. ».

$\Rightarrow$ Ces trois derniers cas correspondent à quelques sites peu nombreux mais produisant l'essentiel des volumes d'eau conditionnés,

- des eaux issues du « socle ", dont les trajets peuvent être très variables, et dont la protection peut être très diverse et parfois incertaine.

$\Rightarrow$ Le plus grand nombre de gisements d'eaux actuellement embouteillées se situe dans ce dernier cas.

Par ailleurs, des trajets courts, des pollutions reçues au stade terminal, des contaminations possibles par des défauts de conception ou des défectuosités d'ouvrages sont également possibles, à peu près dans toutes les configurations dites "protégées ».

\section{CONCLUSION}

Si a priori l'on peut penser, d'une façon quasi restrictive que sur l'ensemble des sites d'eau minérale embouteillée en France, certains devraient pouvoir satisfaire aux contraintes imposées pour des ressources ultimes, on s'aperçoit à l'analyse qu'il n'existe pas de critère de réponse simple, qu'il manque certaines informations pour pouvoir atteindre le niveau de certitude requis, et que si ce type de ressource en eau mérite bien d'être considéré, son identification précise mérite une démarche spécifique.

Réciproquement de nombreux aquifères bien protégés du risque «N.B.C. » - certains parfois très profonds ne satisfont pas aux exigences des eaux embouteillées ou minérales.
Pour les eaux de table et de source, beaucoup de nappes profondes présentent un ou plusieurs paramètres péjoratifs qui ne les rendent pas potables (et que la législation ne permet pas en général d'éliminer). D'autres eaux profondes sont souvent simplement trop minéralisées, ou encore ne sont pas de goût agréable, et ne sauraient être commercialisées. Ces eaux, utilisables en circonstances exceptionnelles, ne pourraient être labellisées « eau de table » ou " eau de source». De même, des eaux minéralisées justifiant l'appellation « eau minérale », utilisables de manière temporaire en secours, ne pourraient que difficilement obtenir les autorisations administratives leur conférant cette appellation (longueur de la procédure de démonstration des propriétés thérapeutiques). En effet, pour les eaux à vocation minérale ou thermale la procédure d'agrément passe par la reconnaissance de propriétés thérapeutiques, longues à démontrer... il est alors plus facile de capter et de redévelopper des sites anciens encore disponibles que de créer ex-nihilo un nouveau produit.

\section{En résumé,}

- certaines eaux minérales ou de source peuvent parfaitement être des eaux d'ultime recours, mais réciproquement, la majeure partie des ressources reconnues protégées ne peut prétendre un jour à la commercialisation,

- préciser lesquelles, parmi les eaux minérales, pourraient jouer ce rôle en toute sécurité exigerait des investigations spécifiques et détaillées sous l'angle non plus seulement du captage, mais du "système de ressource" global, c'est-à-dire du gisement. 


\section{Questions après l'exposé de M. Canel.l.AS}

M. Thirriot (Université de Toulouse) : M. CANELl_as peut-il nous dire si des autorisations ont déjà été délivrées suivant le décret de 1989 compte tenu des délais d'examen qu'il a évalué grosso modo cinq ans ?

Combien y a-1-il eu d'autorisations délivrées depuis 10 ans. depuis 20 ans ?

Quelle proportion cela représente-t-il par rapport au nombre des sites en exploitation depuis les Romains?

1. Canfl.1 As: il m'est difficile de répondre sur le plan national en ce qui concerne les autorisations données depuis 1989 pour l'exploitation d'eaux minérales naturelles. Le décret du 6 juin 1989 concerne les eaux conditionnées, embouteillées mas l'on ne dispose pas encore d'un décret concernant les eaux minérales. Dans le sudouest. quelques autorisations ont été accordées ces dernières années. Je ne connais pas d'autorisation donnée dans le temps record de trois ans.

M. TRICARD : on a une base réglementaire de principe de reconnaissance de l'eau minérale naturelle qui date de textes mis à jour en 1957

Normalement, les autorisations données sur une source d'eat minérale sont d'une durée de trente ans. Le décret de 1989 porte su le probleme des eaux embouteillees uniquement et concerne done un usage particulier. Pour les autorisations nouvelles ou les renouvellements, la durée de la procédure est longue mais on veut avoir une certitude sur la stabilité de l'eau et sur les effets favorables sur la santé

M. Cazevave : les eaux naturelles sont-elles toutes potables et si elles ont des propriétés thérapeutiques, doivent-elles être considérées comme un médicament?

M. Canfllas : elles ne peuvent pas être considérées comme médicament dans la mesure où elles sont conditionnées comme : l'émergence. Lorsque l'on regarde les normes de potabilité, les eaux de sources ne doivent pas franchir les seuils définis pour la potabilité microbiologique. Mais les normes de potabilité chimique ne doiven pas être appliquées car ces eaux contiennent des éléments qui fon leurs propriétés.

M. Ebrard : les délais nécessaires gagneraient à être réduits. Mai il y a des autorisations provisoires qui ne semblent pas avoir de caractère réglementaire.

M. TricarD : s'il n'y avait pas de doutes sur la stabilité de l'eau. on pourrait réduire le temps de procédure. Mais on pousse les responsables de dossiers à faire des analyses de suivi pour voir la gamme de fluctuations naturelles de l'eau. Pour l'autorisation provisoire, elle $n^{\prime}$ 'a pas de fondement juridique sûr: il y a des réflexions actuellement sur la possibilité de poursuivre dans cette voie.

Intervention de René Troulı.ı. (division des eaux minérales du ministère de l'industrie)

Concernant les délais, il y a deux procédures qui sont très longues dans le temps : la préparation du dossier car il s’agit de prouver que l'on a bien affaire à une eau minérale du point de vue physique. ce qui demande un certain nombre d'examens étalés sur plusieurs mois voire des années pour prouver que la composition de l'eau est bien constante aussi bien du point de vue physico-chimique que bactériologique. Lorsque le dossier est prêt. le traitement du dossier entre le moment où il est déposé chez le préfet et le moment où il est présenté au conseil départemental d'hygiène et adressé au ministère es de l'ordre de quelques mois seulement.

Il faut quand même deux analyses faites par l'agence du médicament à six mois d'intervalles, c'est la réglementation qui le prévoit.

\section{Question de M. Lugan (société Christ)}

Il faut distinguer dans le contexte européen les deux sortes d'eaux : les eaux en bouteille et les eaux utilisées dans les établissements thermaux. Ces dernières ne sont soumises à aucune disposition communautaire. En ce qui concerne les eaux en bouteille, une directive de 1980 a pour objet de faciliter la circulation des eaux en bouteille. Cette procédure nécessite l'envoi à la commission européenne d'une liste régulièrement mise à jour des eaux reconnues comme minérales naturelles dans l'état membre. Le cas des eaux venant de l'extérieur de la CEE. est différent : il faut que les eaux passent par un pays qui décidera si oui ou non il faut inscrire cette eau sur lit liste européenne.

Intervention de M. Avıs, géologue conseil de N.S.I. (g.g.s Source Perrier).

Je voudrais seulement signaler que la Loi française de 1989 sur les eaux minérales, en imposant certains changements d'étiquettes alor que l'étiquetage antérieur avait été officiellement (depuis 1978 accepté aux Etats-Unis, a entrainé une réaction du public américain qui a fait chuter la consommation de l'Eau Perrier de plus de $40 \%$ aux U.S.A.

Le public américain (après l'affaire du Benzène également ma présentée. car due seulement à ce que la norme américaine pour le Benzène, a été placée à $50 \%$ de la norme française qui elle était respectée !) n’a pas accepté qu'une eau jusqu`alors étiquetée " eat minérale gazeuse naturelle" devienne " eau minérale naturelle enrichie au gaz de la source » et a estimé qu'il avait été antérieurement trompé par Perrier

Or le mot enrichi (cf. mes rapports de l'époque) n'est pas justifie. car il laisse croire à un enrichissement artificiel en gaz. En réalité la réintroduction de gaz à l'embouteillage a pour but de maintenir la teneur en ga: de l'eau commercialisée, à exactement celle de l'eau de la Source Perrier originelle, qui a indiscutablement été démontrée comme étant de 3 à 3,5 volumes par litre, fait officiellement accepté par les Etats-Unis après examen approfondi du problème en 1978 Los Angeles. Personnellement. je regrette que mes avis hydrogéologiques, contrairement à ce qui avait eu lieu en 1978, n'aient pas été pris en compte pour les décisions finales de changement d'étiquette.

\section{Question 1 sur le rapport Ebrard}

Avec le rapport de 1980-81, un certain nombre de points ont été acquis: l'attestation d'hydrologie a été transformée en capacité de médecine ; deuxièmement la prise en charge du curiste. la procédure de l'entente préalable a été modifiée, le délai de 3 mois laissé à la sécurité sociale pour répondre et dont le silence valait refus a été transformé en un délai de 21 jours et dont silence valait acceptation. Il avait enfin été acquis et publié que l'enseignement de l'hydrologie était rétabli au concours de l'internat de médecine. Obligation pour les étudiants de recevoir un enseignement en hydrologie, le programme de l'internat étant celui du cursus du deuxième cycle des études médicales. Malheureusement la loi hospitalière qui politiquement avait été adoptée 3 ou 4 années plus tard a supprimé cette mesure et l'a annihilée. Parmi les propositions d'aujourd hui on reprend ce qui malheureusement avait été supprimé et qui doit être rétabli c'est-ìdire un enseignement obligatoire de l'hydrologie et deuxièmement après cette phase intermédiaire qui avait amélioré l'entente préalable. nous proposons sa suppression pure et simple. Je ne veux pas anticiper sur les mesures qui seront prises. Donc cet engagement de ne pas arrêter le remboursement des cures par la sécurité sociale ouvre une période de stabilité du thermalisme. Ce qui ne veut pas dire pour autant. que l'avenir. lui. est protégé. Il faut que le corps médical, que les professionnels en liaison étroite avec l'administration dans une collaboration bien comprise et dans un partenariat bien senti arrivent les uns et les autres à maîtriser le développement du thermalisme.

Intervention de M. Frifs (Thermes de Luchon)

J'ajoute une petite précision concernant les remboursements par la sécurité sociale : ils sont passés de $70 \%$ à $65 \%$ en août 1993.

\section{Intervention de Henri Ussov (Figaro)}

Quels sont les obstacles qui s'opposent encore au développement de la remise en forme en milieu thermal. La remise en forme non remboursée par la sécurité sociale représente environ 25000 clients par an. La thalassothérapie a développé un marché de 135000 curistes libres. Quels sont actuellement les obstacles réglementaires qui s'opposent au développement de la remise en forme en milieu thermal?

\section{Réponse}

En tant que médecin de la sécurité sociale je peux dire que rien ne 'oppose à la remise en forme si ce n'est que la clientèle n'étant pas remboursée boude les centres de remise en forme.

\section{EBrARD}

Je citerai simplement un exemple : la remise en forme et la détente font largement appel aux piscines. Or la réglementation des piscines thermales interdit qu'elles soient purifiées alors que les piscines 
publiques doivent l'être faute d'être fermées. Dans cette distorsion où chacun d'ailleurs a ses arguments, il y a simplement une difficulté majeure qui doit conserver le bénéfice thérapeutique d'une eau pour les malades alors que ceci n'est pas exigible pour des bien-portants. A vous d'apprécier comment la réglementation peut être adaptée et ce n'est pas facile. Comment peut-on ouvrir des établissements à une clientèle jeune d'accompagnants ou à une clientèle ludique si les piscines ne peuvent pas leur être ouvertes dans des conditions qui en facilitent l'usage ? En Allemagne et Italie, la clientèle des stations thermales est plus importante qu'en France car elle utilise les eaux pour la détente et le côté ludique. Les séjours sont souvent plus courts.

Question de Jean-Louis BUrEAU. (Président de l'UNAT)

D'une part pour ce qui est de la thalassothérapie, loin de moi l'idée de les critiquer, je pense que c'est également une thérapeutique reconnue et ils ont fait leur trou en France plus particulièrement. Parce que le thermalisme n'a pas pu ou su développer des cures à durée variable comme d'ailleurs cela a été proposé dans le dernier rapport Ebrard. Simplement qu'est-ce que c'est d'abord que la remise en forme? En clair: ce sont des soins qui ne sont pas remboursés, ni plus ni moins: l'on n'invente rien. Dans les différents centres de thalassothérapie, de thermalisme en France, en Europe, on trouve les mêmes baignoires, les mêmes fournisseurs, les mêmes douches et pas forcément et fort heureusement les mêmes eaux caractéristiques de chaque station ? Il s'agit donc là de remboursement et seulement de cela. Puisque la remise en forme je crois que les stations thermales en font depuis toujours.

\section{Communication de Messieurs Canellas et Blavoux}

\section{Communication de M. PouchaN}

Question de Mme FABRI (maître de conférence faculté de médecine de Clermont-Ferrand)

Par quelle méthode avez-vous mis en évidence l'âge des sources thermales?

Réponse : C'est un travail réalisé en équipe avec M. Bl.Avoux. Les financements viennent tantôt d'ELF, tantôt de Gaz de France. C'est la méthode du Carbone 14 qui est utilisée. On a mis au point la méthode, plus rarement employée en France, des gaz rares en solution. Lorsqu'une eau s'infiltre elle possède une goutte de pluie qui, à une altitude déterminée, possède un stock de gaz en solution et parmi les gaz en solution qui sont présents dans l'air, il y a les cinq gaz rares de l'air. Cette quantité de gaz dissoute est fonction de la pression et de la température du lieu. Comme ces gaz sont inertes, et qu ils ne réagissent pas en principe avec le contexte physique souterrain, ils conservent donc une mémoire des conditions qui régnaient au moment de l'infiltration. A ce moment on va les pêcher avec une source ou un forage, on peut, toujours si on connaît l'âge, décrire le climat qui régnait au moment de l'infiltration, et si on a une courbe des climats donner l'âge de l'eau.

Question de M. Michel LARoque (Compagnie générale de géophysique)

Au sujet des granites, vous semblez un peu sceptique sur des possibilités de fracturation très profonde. Je pense qu'ils sont mal connus, étant donné que les pétroliers les ont très peu étudiés, vu que pour eux, ce n'était pas prioritaire. On commence à les étudier avec des moyens importants pour les futurs stockages des déchets nucléaires, etc... On se rend compte à l'aide des études récentes qu'effectivement, en géophysique, on arrive à distinguer des failles très importantes qui peuvent faire d'une centaine de mètres jusqu'à 5000 mètres de profondeur.

\section{Réponse de M. PouchaN}

On ignorait tout cela jusqu'à maintenant. Ce que j'ai évoqué c'est la fracturation par décompression. J'ai du mal à imaginer qu'à 5000 mètres de profondeur on puisse avoir une fracturation par décompression.

Réponse de M. Jacques Avias (Université Libre du Vivarais)

En ce qui concerne la décompression génératrice de fissures ouvertes dans les roches rigides elle comprend:

- la décompression superficielle, due à la proximité de l'atmosphère, elle se fait sentir sur 30 à 50 mètres sous la surface du sol,
- les décompressions d'origine tectonique s.s. ou globotectoniques dues aux mouvements orogéniques ou épiorogéniques, ou aux mouvements des "plaques \%. Ces décompressions peuvent être beaucoup plus profondes.

A titre d'exemple. à l'occasion d'études de sites de barrages dans les Cévennes, ont pu être observées, dans des sondages, des fissures ouvertes plus profondes que celles correspondant à la décompression superficielle. Ces fissures sont liées à l'effondrement du graben de la vallée du Rhône (faisant partie de la grande cassure de la plaque européenne qui va d'Espagne en Norvège en passant par le fossé Bressan et le fossé Alsacien) ; effondrement confronté à l'Ouest à la surrection du a Hot Spot s (point chaud) du Massif Central. L'effondrement différentiel correspondant explique la fissuration tant du socle hercynien que de sa couverture sédimentaire (notamment calcaires karstifiés de piedmont). Enfin il est nécessaire comme M. PouCHAN le souligne que l'on ait une * tectonique vivante " sans laquelle très rapidement les fissures ouvertes se colmatent, de silice, ou de calcite, etc... Une telle tectonique vivante existe dans la zone cévenole et dans les karsts languedociens et explique la richesse en eaux souterraines de ces derniers, comme elle explique en Italie l'existence des célèbres sources karstiques du Latium (contiguës à la cassure de plaque sur laquelle se trouve le Vésuve): source dont le débit d'étiage exceptionnel $\left(63 \mathrm{~m}^{3} / \mathrm{seconde}\right.$ au total $)$ peut expliquer en partie le développement de la civilisation romaine.

Question de Mme Francine MerciER (groupe BRGM)

Il ne faut pas pousser les gens à faire de la mesure pour faire de la mesure car on ne peut pas l'interpréter. Il faut choisir une source que l'on connaît. savoir si elle a des mélanges, connaître ces mélanges. Mais si l'on pousse les gens à ne pas faire de mesures, ils n'auront pas de chronique, d'historique. L'historique me paraît indispensable.

M. BLAvoux : les isotopes interviennent à deux niveaux : quand on n'a pas de données, pour débrouiller un problème ; ensuite, c'est performant pour le suivi. L'investissement n'est pas excessif par rapport à un forage.

M. LOPOUKHINE : le BRGM m'a entrepris pour collecter les informations disponibles sur le passé et les compléter sur certains sites par des mesures pendant la méthode du tritium et par des mesures nouvelles pour constituer des séquences ou chroniques $3 \mathrm{H}$ sur certains sites.

M. Langguth : Dans l'Eifel et dans les Ardennes la teneur en $\delta{ }^{13} \mathrm{C}$ a été déterminée sur plus de 400 échantillons. Ces valeurs se concentrent autour de -6 a $-5 \delta{ }^{13} \mathrm{C}$. Un certain nombre de dosages ${ }^{4} \mathrm{He}$ a montré que toutes les valeurs ne sont pas d'origine profonde, sauf celles avec des teneurs élevées en ${ }^{4} \mathrm{He}$, même si celui-ci reste en dessous de $10 \%$ de l'hélium total. Ces critères favorables pour une origine profonde sont aussi la situation géologique en zone de grandes failles et une teneur plus élevée en $\mathrm{Cl}^{-}$.

M. Blavoux : je suis d'accord. On a des preuves de venue de chlorures profonds dans des socles volcaniques mais aussi cristallins. Il faut des accidents très profonds (plusieurs milliers de mètres).

M. Jambac (Géoprospect) : quand on parle de l'âge de l'eau, on a l'impression que l'eau est une masse poussée vers l'émergence. Or on voit que le volume d'eau qui sort est un mélange d'eaux avec des temps de transfert différents.

M. BLAvouX : quand on parle d'âge, c'est un âge moyen. Dans le cas d'Evian, c'est un temps de filtration. On fait un traçage par exemple avec de la fluorescéine. Le tritium va continuer à sortir pendant de très nombreuses années. Un modèle piston voudrait qu'on ait propagation des eaux depuis la zone d'alimentation jusqu'à l'émergence avec très peu de mélanges. Je n'y crois pas beaucoup. C'est plutôt un mélange.

M. Jambac : dans ce cas, il y aurait des eaux qui n'auraient que quelques années.

M. Blavoux : non car si on avait des eaux qui ont quelques années, on aurait du tritium, or il n'y a pas de tritium dans les systèmes anciens qu'on étudie. Il y a un marquage paléothermique. Des eaux se sont infiltrées avec les gaz rares à une température de $6{ }^{\circ} \mathrm{C}$ alors qu'elles sont actuellement à $11^{\circ} \mathrm{C}$. On est sûr de ce temps de séjour minimum de 18000 ans. 
M. Jамвас : et quand une eau remonte à la surface, elle recoupe d'autres aquiferes. A ce niveau-là, qu'est-ce qu'on peut faire ?

M. BLavoux : c'est le problème des mélanges à l'émergence. entre le flux principal de la profondeur et les venues en sub-surface. La chimic et les isotopes permettent de bien reconstituer les proportions du mélange.

\section{Exposé de M. Gadi.ia}

Question de M. Thirriot : Jusqu’à quelle profondeur vont les bactéries? La contamination se fait-elle après l'émergence ou bien est-ce les eaux météoriques qui entrainent les bactéries?

Réponse de M. Frifs (Thermes de Wermon) : La présentation de Monsieur GADALIA résulte de travaux réalisés sur les sources de Wemon. en 1984. Depuis cette époque une campagne de forages a été conduite, et actuellement les sources ne sont plus utilisées. et les thermes sont alimentés exclusivement par 2 forages réalisés dans les zones de sources repérées Nord et Sud. Ces forages, respectivement a $133 \mathrm{~m}$ et $160 \mathrm{~m}$ (Nord et Sud) permettent de disposer d'eaux sulfurées-sodiques à $65^{\circ} \mathrm{C}$ et $74^{\circ} \mathrm{C}$

Les effets de "variations saisonnières " observées lors de l'étude des sources par hydrogéochimie étaient liés aux variations de pression hydrostatique des couches superficielles chargées en eaux pluviales à des taux variant selon les saisons et la pluviométrie qui les caractérisent.

En ce qui concerne les bactéries pathogènes, il s'agit de pollutions extérieures qui viennent soit de ce que l'on a introduit dans le gisement soit des moyens employés dans le gisement soit du forage et des matériaux utilisés ou ensuite des circuits de distribution et de stockage des eaux avant utilisation aux postes de soins. C'est là-dessus qu'il faut être particulièrement vigilant car les pollutions se réalisent très rapidement et on finit par avoir dans certaines zones des nids bactériens qui sont très difficiles à résoudre et vous avez parlé également des biofilms qui sont eux aussi très résistants.

\section{Réponse}

La question concernait principalement les bactéries chimio-litotrophes dont certaines appartiennent au cycle du soufre. On sait que ces bactéries ont des possibilités d'adaptation extrêmement grandes. Elles proliferent en particulier dans des milieux qui sont très acides avec des températures très élevées. Les chimio-organotrophes ne peuvent pas se développer dans des milieux au $\mathrm{pH}$ aussi élevé.

\section{Honegger (Société ANTEA groupe BRGM)}

On note la présence de facteurs sulfatoréducteurs en fond de puits géothermiques à plus de $2000 \mathrm{~m}$ et $70^{\circ} \mathrm{C}$. Par ailleurs ces bactéries ont été trouvées dans les rifts médico-océaniques à des profondeurs de $2000 \mathrm{~m}$ et $140^{\circ} \mathrm{C}$.

Précision sur les bactérie du soufre : celles-ci sont présentes dans l'eau à l'état " de stress nutritionnel " c'est-à-dire qu'elles remontent à la nuit des temps. Elles subissent des pressions et des températures très élevées et sont enkystées. Lorsqu'elles arrivent dans une zone de multiplication favorable, elles ne demandent qu“à se régénérer.

\section{Question de M. de MarsiL)}

Quel est l'éventuel effet positif de la présence de bactéries non pathogènes dans l'eau embouteillée ? Un des points importants, pour la préservation des eaux embouteillées c'est qu’à partir du moment où elles sont ouvertes elles résistent un certain temps à des contaminations éventuelles. Est-ce que la présence d'une colonie de germes non-pathogènes peut aider à préserver du développement de germes pathogènes ?

\section{Réponse de Mme PEPIN}

Nous avons mené une expérience sur le Pseudomonas aeruginosa en tant qu'indicateur. Nous avons ensemencé une eau de surface qui contenait beaucoup de bactéries et par ailleurs une eau pratiquement dépourvue de bactéries. Au bout de quelques années les bouteilles ont été réouvertes. Celle qui contenait de l'eau de surface n'en contenait pratiquement plus alors qu'ils avaient pullulé dans l'eau qui ne contenait pas de bactéries. Par conséquent, cela dépend des bactéries à qui on a affaire mais il faut savoir qu'il y a des équilibres dans certains écosystèmes. et que certaines bactéries sont susceptibles de sécréter des antibiotiques.

\section{Remarque de Mme BoвET}

La circulaire du 29 juillet 1992 ne concerne que le thermalisme et. en particulier, les critères de qualité relatifs aux legionella ne sont pas applicables aux eaux embouteillées, le risque sanitaire représenté par la présence de ces germes étant lié à l'inhalation d'aérosols contaminés et non pas par ingestion d'eau.

\section{Remarque de M. Derain}

La loi sur l'eau va permettre d'inclure des mesures de protection du bassin d'alimentation, dans les périmètres de protection.

Mais ces mesures ne peuvent porter que sur les usages futurs. Si l'on veut toucher à l'existant. il faut indemniser. Dans beaucoup de cas, cela serait politiquement et financièrement difficile. II faudrait commencer par les gisements à faible durée de circulation de l'eau.

Remarque de M. BLAvoux sur les problèmes de délimitation des bassins d'alimentation.

Cette délimitation n'est pas si facile et d'autre part sur ces bassins il existe une activité. Si on la neutralise, il reste le problème de l'entretien. ce qui pose un problème de gestion. II faut penser aux générations futures : il faut laisser une eau correcte, c'est important. et ne pas se retrancher derrière cette histoire de temps de séjour, si vous avez un système qui a un temps de séjour ou un temps de transit de 50 ans, et que la source est polluée, c'est fini.

\section{Remarque de M. Avias}

La réglementation actuelle n'est absolument pas suffisante, car il existe des gens qui achètent des marécages dans le seul but de les revendre à Perrier deux ou trois fois leur valeur. Pour la source du Lez, on aurait pu la sauver mais les périmètres légaux n’ont pas été respectés. Il est donc très important d'avoir des réglementations draconiennes et le bilan économique ne serait pas négatif.

\section{Réponse de M. de Marsily}

Il y a des sources où on ne pourra rien faire. Par contre il y en a où le coût de la protection a contre lui le fait qu'on investit pour dans 100 ans et donc économiquement ça n’est pas possible de calculer sa rentabilité. Si on se limite à des calculs économiques on ne fera jamais rien. Du point de vue national ce n'est pas acceptable il me semble que la notion de parc a un rôle administratif. Il est difficile car il faut préciser la zone exacte d'alimentation. Mais il existe des gens tout à fait compétents. Tout n'est pas protégeable mais si on ne fait rien dans 10,20, 50 ans on n'aura plus d'eau minérale.

\section{Question de M. BARBIER (INRA-SAD}

La protection des gisements d'eau minérale au niveau de l'implevium butte sur un problème de droit: comment privatiser une ressource minérale dont on demande qu'elle soit gérée au niveau local (par exemple en impliquant les agriculteurs) collectivement? Le travail de protection des ressources d'eau minérale va impliquer pour les producteurs d'eau minérale un véritable investissement en terme sociologique pour comprendre les dynamiques locales et permettre une gestion concertée. Le problème n'est pas simplement technique ou juridique.

\section{Remarque de Mme Ot.ivfros-Toro}

Une première réaction en tant qu'hydrogéologue :

La mise en place de zones de protection renforcée dites a réserves hydrogéologiques " ne risque-t-elle pas de faire naitre dans les esprits la possibilité d'être plus “ laxistes " en permettant beaucoup de choses dans les zones en dehors des "réserves"?

La loi sur l'eau prévoit la mise en place de périmètres de protection de la ressource. Toutefois, dans le cas des eaux minérales se pose le problème de l'intervention de l'Etat dans des activités industrielles privées lors de la mise en place de périmètres de protection par des sources déjà exploitées. Il faudra faire la part entre préservation de la ressource et intérêts privés. Le travail en cours au ministère chargé de la Santé. avec la participation des ministères de l'environnement et de l'industrie devra répondre également à cette question.

\section{Question}

En Allemagne vous avez quatre classes d'eau embouteillée. En France il n'y en a que deux : les eaux minérales et les eaux de sources. Pourriez-vous nous expliquer la législation allemande?

\section{Réponse de M. HERCh}

L'appellation " eau de table " recouvre n'importe quelle eau de n'importe quelle origine, même de l'eau de rivière à condition que l'eau corresponde à la loi sur la nourriture. Elle n'est pas soumise à 
la loi de médecine. Tandis que les eaux médicinales ne doivent subir aucun traitement. Pour les eaux minérales naturelles, la loi est la même qu'en France.

Question de M. BLAvoux

Est-ce que vous avez des idées sur la teneur en isotopes du $\mathrm{CO}_{2}$ avant de rejoindre le réservoir?

\section{Réponse}

On pense qu'il y a un acide fort et il est très probable que cet acide fort attaque des calcaires sous la faille eifelienne. Il y aurait à ce moment-là précipité de sulfates et émission de $\mathrm{CO}_{2}$ qui migre vers le haut. Il peut peut-être traverser sans prendre beaucoup de minéralisation, comme à Spa, mais dans le cas de Chevron il rencontre la partie supérieure sur 100 ou 200 mètres et nous pensons que c'est là effectivement que la minéralisation est prise.

\section{Question}

Quel est le temps de séjour des eaux peu minéralisées dans le système minéral superficiel ? Ensuite avez-vous des exemples de remise en état d'un gisement endommagé par l'activité humaine ?

\section{Réponse}

Les eaux ont un délai de circulation de six mois. Les petits barrages dont je vous ai parlé servent à éviter le ravinement. Et on a vu notamment les teneurs en matières organiques et en nitrate qui augmentaient de façon significative. On a dû enlever ces dispositifs. curer les dépôts qui s'étaient sédimentés dedans, et reprendre des dispositifs anti-érosion tout à fait normaux.

\section{Journée du 17 novembre}

Question de M. LeconNet

La compétence scientifique présentée sur la maîtrise des eaux carbogazeuses apparaît complète et permet de lever tous les problèmes technologiques dans leur exploitation. L'exploitation des eaux en géothermie pose des problèmes analogues. Quel est votre sentiment sur la compréhension et la maîtrise acquise aujourd'hui qui devraient éviter les déboires apparus dans beaucoup de réalisations mises en place il y a 20 ans et pour lesquelles les phénomènes de base ne semblent pas avoir été suffisamment pris en compte.

Réponse

Les problèmes techniques en géothermie ont été un moment sousévalués mais actuellement on a pu développer de nombreux logiciels de modélisation et de connaissances techniques d'exploitation. Je voudrais donner une des origines des problèmes d'exploitation de la géothermie ; ils sont beaucoup plus liés à un problème de maitrise d'œuvre de ces projets car il y a une séparation très précise entre l'hydrogéologue et les gens qui ont fait l'équipement de l'exploitation des ouvrages.

\section{Question de M. Pouchas}

Beaucoup d'eaux de source minérale ont reçu un agrément d'après leur composition chimique à l'émergence. Or il se trouve que les équipements modernes conduisent souvent à une modification de cette composition. Comment faire pour relier ces deux aspects ?

\section{Réponse}

La position donnée par l'académie de médecine est que si il y a une eau dont les qualités physico-chimiques s'écartent de plus de $20 \%$ de ce qu'elles étaient, alors il y aura de nouveaux essais pour justifier les propriétés de l'eau. A l'exploitant de prouver que son nouveau captage donne une eau qui a les mêmes propriétés que les eaux précédentes. Ceci pour éviter un certain nombre de débordements et des exploitations sans aucun but thérapeutique.

\section{Question de M. Pouchax}

Quel est votre point de vue sur les effets du radon?

\section{Réponse}

On n'a pas beaucoup d'information sur l'activité du radon. Ce n'est pas un paramètre qui est pris en compte chez nous. Pour ce qui est de la radioactivité des eaux, on ne met plus l'accent dessus depuis quelques décennies. On doute fort de l'efficacité de la radioactivité, la seule chose c'est que l'académie de médecine est de plus en plus restrictive sur son utilisation.

\section{Question de M. JEHI}

La méthode radon est-elle adaptée à tous les types de terrains géologiques?

\section{Réponse}

L'utilisation de la méthode du radon n'est pas toujours adaptée et n'est pas systématique. Pour des gisements ou des terrains très profonds on arrive à avoir des émanations de radon ou de $\mathrm{CO}_{2}$ en surface. La grosse difficulté est que le fait d'avoir des anomalies ne permet pas d'affirmer que l'on va trouver l'eau minérale désirée. Alors doit-on s'implanter sur un indice? Tout dépend du type de fissurations. C'est du cas par cas. Le radon est un outil parmi d'autres.

\section{Remarque de M. Trouil.ier}

La prospection par radon est un outil parmi les autres, et il convient de confronter les résultats de toutes les autres méthodes (géophysique, prospection électrique, sismique, gravimétrie, etc...) pour déterminer un ensemble cohérent de probabilités.

Remarque de M. LOPOUKHINE

La méthode du radon présente un certain nombre de limitations. Premièrement trop séduisante et trop facile à mettre en cuvre. Quand sur une carte apparaissent des anomalies rouges, ça déclenche immédiatement un réflexe de spéculations foncières, pour ensuite se réserver la possibilité d'y faire un forage.

Deuxièmement, c'est une méthode qui marche trop bien. Le radon marche aussi d'une façon parfaite pour trouver des sources d'eau potable en Bretagne. Quel que soit le type d'eau que l'on cherche on aura une réponse. Le problème particulier posé par les eaux minérales est de savoir comment différencier un signal que l'on recevra de toute façon. Il est déjà arrivé de trouver de l'eau potable là où on s'attendait à trouver de l'eau minérale.

Question de M. LANGguTH

1) Faut-il faire confiance aux anciennes analyses ?

2) Connaissez-vous des études concernant le fractionnement isotopique du soufre appliquées à votre schéma d'oxydation.

\section{Réponse}

Il faut savoir absolument comment ces analyses ont été faites. En général c'est par iodométrie. C'est valable en ce qui concerne la somme du soufre réduit total mais par contre pour ce qui est de différencier le soufre -2 et le soufre +4 , il ne faut absolument pas faire confiance aux anciennes analyses. De plus, les résultats sont d'autant plus erronés que l'eau est chaude.

Question de Mme Chaigneal

Avez-vous déjà effectuer des dosages des sulfures en chromatoionique?

\section{Réponse}

Non bien que nous ayions le matériel au laboratoire. Il semble qu'il y ait des publications qui commencent à apparaître mais elles ne permettront pas en une même analyse de faire un fractionnement des dérivées comme c'est possible avec la potentiométrie. Par ailleurs, toutes les méthodes de chromatographie ionique qui utilisent des diluants tamponnés $\mathrm{pH}$ peuvent modifier considérablement la répartition des espèces. L'autre problème est que ce n'est pas transposable sur le terrain.

\section{Journée du jeudi 17 novembre, après-midi}

Exposé de M. Mialonier (réponses suite à sa communication) Exposé de M. TeysSEYre

Question de M. Boulange

L'éradication des maïs tient plus à des pollutions par les nitrates ou la trazine?

\section{Réponse de M. MiALONIE.}

Le plus gros risque est lié à l'absence de toute végétation pendant une période importante au risque de lessivage des nitrates.

\section{Réponse de M. TEYSSEYRE}

On ne se pose pas ce type de question. On a voulu la stopper avant qu'elle prenne une plus grande importance.

En ce qui concerne le réseau routier, l'un des problèmes majeurs est en zone montagneuse, le salage des routes, mais des mesures 
simples par exemple l'utilisation de saumure peut permettre une diminution importante des quantités utilisées pour une efficacité identique. On peut minimiser l'apport avec une efficacité identique.

\section{Exposé de M. Mesny}

\section{Question de M. Cazenave}

Après avoir exposé tant de raisons d'inquiétudes, pouvez-vous esquisser des solutions permettant d'espérer?

\section{Réponse}

Un homme averti en vaut deux. Il vaut mieux avoir réfléchi à ce genre de crise même si elle a une probabilité excessivement faible et faire quelque chose lorsque la crise arrive. Les problèmes ne sont pas uniquement d'ordre nucléaire, ou biologique. Nous sommes dans une civilisation de plus en plus sophistiquée, et il suffirait d'un grain de sable pour que la machine s'arrête. Du point de vue eau potable nous avons un système l'un des plus sûrs au monde. L'incident de Milwaukee ne pourrait pas se produire en France en particulier parce que on surveille de très près la turbidité de l'eau. La ville de New York par exemple ne traite pas son eau. Elle la prend directement depuis un barrage en y additionnant beaucoup de chlore. Nous avons donc toutes les raisons d'être optimistes mais il faut rester attentif.

\section{Question de M. BLAvoux}

Concernant des précisions sur le temps de trouver de l'eau d'Evian, je renvoie au texte de ma communication et rappelle que le temps minimum de filtration s'élève à 16 ans et que le temps moyen de séjour dans l'aquifere a été estimé à 50 ans compte tenu de la dispersion du tritium entrant dans un réservoir de grand volume.

\section{Réponse}

Je vous ai présenté une étude nationale et la prochaine étape, qui est confiée aussi au BRGM c'est de faire l'application de ceci et de préciser les forages existants ou à installer. Sur deux zones en particulier, la région parisienne et la région lyonnaise, se portera l'étude.

\section{Intervention de M. DERAIN}

Cas de Volvic : un circuit plus rapide peut effectivement exister au contact granite-pouzzolane de l'ancienne vallée comblée.

Actuellement, pour les forages de remplacement, on évite d'aller jusqu'à ce contact, de façon à éviter au maximum le prélèvement de cette eau plus jeune.

Question de M. Blavoux à M. Mesny

Pensez-vous aller plus loin que la réflexion sur la vulnérabilité des aquifères en encourageant les interconnexions de réseaux ou en mettant en place des captages de recours ? En cas d'accident on ne peut concevoir ces réalisations en quelques heures ni même en quelques jours.

\section{Réponse}

L'inventaire des captages a été confié au BRGM. On va voir en région parisienne quel est l'état des forages existants et prévoir éventuellement la construction d'autres forages. Le deuxième point important est la ville de Lyon qui tire l'essentiel de son eau potable du Rhône : il faut pouvoir exploiter des forages.

Commentaire de M. Plaud (Agence de l'Eau Adour-Garonne)

Il existe une douzaine de forages captant l'eau de nappes captives donc bien protégées des pollutions de-surface voire atmosphériques qui sont maintenus prêts à fonctionner en cas de pollution nucléaire à l'aval de Golfech (à partir de la centrale de Golfech) de l'eau de la Garonne utilisée par certaines collectivités.

Ces forages ont été financés par EDF.

M. MesNy : il faut faire attention aux installations de secours qui ne fonctionnent pas.

\section{Jacques Avias :}

M. de MARSILY hier a insisté sur la pollution constatée dans de nombreuses nappes, à partir d'éléments infiltrés provenant de rejets de stations d'épuration ou de rejets de fumées polluées d'usines industrielles ou d'usines d'incinération d'ordures ménagères.

A titre d'exemple. les rejets des stations d'épuration des eaux du bassin d'alimentation de la source du Lez, ont montré (par des études avec les isotopes de l'azote) que certains éléments avaient déjà atteint la presque totalité des aquifères, mais en quantité encore extrêmement faible par rapport aux normes. Le problème pour le futur est celui du cumul possible avec le temps.

Un autre exemple est celui de l'usine d'incinération des ordures ménagères de Lavilledieu près d'Aubenas dans l'Ardèche, par 36 communes, four allumé en 1986 mais qui a dû être arrêté en 1992 vu le constat des nuisances sur l'environnement (plus acide chlorhydrique) ayant provoqué par exemple en moins de 3 ans la libération en moins de 10 hectares de $35 \mathrm{~kg}$ de zinc dans le sol et les eaux à partir de la galvanisation des clôtures. Ce sans compter la mise en décharge des scories et cendres des fours en décharge.

Réponse : c'est en effet un problème important car on fait de plus en plus appel aux eaux de surface.

Commentaire de M. Ricour : la ville de Marseille est également alimentée par des eaux de surface.

Question de M. GIRAud (SAGEP - Eau de Paris)

Quel est le volume d'eau embouteillée stocké ? et quelle est la capacité de production des usines d'eau embouteillée ?

Réponse : la production d'eau embouteillée de l'ordre de 5 milliards de litres par an, la production est quasi continue. On ne peut pas demander de doubler la capacité de production. En plus, un milliard de litres est pour l'exportation. De plus, des limites sont dues aux emballages. 
Neyrpic Grenoble - Stages constructeurs

Thème : Régulation et Automatismes.

Ces stages personnalisés concernent :

- les principes et méthodes de la régulation de vitesse des groupes hydrauliques et thermiques (étages électroniques et hydrauliques):

- les divers produits de la gamme des régulateurs Neyrpic, leur exploitation et leur maintenance.

Ces formations peuvent être dispensées dans les locaux de Neyrpic ou chez les clients.

Renseignements: Direction Régulation-Automatismes, Neyrpic, 75, rue Général Mangin, BP 75 Grenoble Cedex. Tél. : 76393000 (poste 3870), Télex : $320750 \mathrm{~F}$, Télécopie : $76 \quad 393455$.

\section{CAST (stages)}

Le CAST (Centre d'actualisation scientifique et technique), organise de nombreux stages de formation continue tout au long de l'année.

Renseignements : CAST, INSA, Bâtiment 705, 20, av, Albert-Einstein, 69621 Villeurbanne Cedex av. Albert-Einstei
Tél. : $78 \quad 93 \quad 24 \quad 45$.

\section{CEFIGRE (sessions de formation)}

Organisation: Sessions organisées, soit à Sophia-Antipolis, soit dans divers pays d'Afrique ou d'Asie.

Thèmes: Tous problèmes relatifs à l'approvisionnement en eau en milieu urbain ou rural Pour tous renseignements détaillés sur les programmes des différentes sessions, voir cidessous.

Renseignements: CEFIGRE, Sophia-Antipolis, BP 13,06561 Valbonne Cedex, TÉl. 16/93 74 31 00, Télex : 4613 11 F.

\section{Centre de formation permanente VERSEAU, Montpellier (séminaires)}

Thèmes: Nombreux séminaires de quelques jours concernant: les eaux usées, l'hydrologie, le traitement et la distribution des eaux.

Renseignements: M. J.C. Le Gars, CREUFOP, 99 av, d'Occitanie, 34075 Montpellier Cedex, Tél. 67634803 . Télex: $490944 \mathrm{~F}$.

\section{Centre international de l'eau de Nancy (stages)}

Thèmes : Eau et environnement : Eau et informatique ; Eau et génie industriel.

Renseignements: Centre international de l'eau (Responsable Formation: Patrick Faivre), 149, rue Gabriel-Péri, 54500 Vandoenure, Tól. : 835664 Télex: $960753 \mathrm{~F}$

\section{Formation instrumentation}

Organisation: Sté Endress + Hauser.

Thèmes: Mesures de débit, de pression, de niveau sur fluides et solides. Mesures physico-chimiques, d'humidité et de température.

Renseignements: M. G. Eckenschwiller (Tél. 89696768 , poste 741 ). Endress + Hauser, BP 5. 68330 Huningue (France).

\section{Water training courses}

Thèmes: 1) Management and Supervisory Development ; 2) Water Law and Finance ; 3) Operations ; 4) Engineering ; 5) Water Science : 6) ICA and Information Techno$\operatorname{logy}$; 7) Health and Safety ; 8) Understanding the Water Industry.

Renseignements: Marketing and Development Section, Water Training, Tadley Court, Tadley Common Road, Tadley, Basingstoke, Hampshire RG26 6TB (Royaume-Uni).

\section{IHE, Delft (Pays-Bas)}

Cet institut propose des formations diplômantes dans les domaines de l'eau, de l'environnement et des transports ainsi que des cours.

Thèmes des formations diplômantes : hydraulic engineering with four specialisations (river engineering, coastal, estuarial- and harbour engineering, land and water development, hydroinformatics), hydrology and water resources (hydrological processes, groundwater hydrology, water resources management).

Thèmes des cours diplômants : environmental science and technology, water quality management.

Thèmes des séminaires: management of the coastal environment in the hydroinformatics era, port management, appropriate modernization and management of irrigation systems, integrated coastal zone management, anaerobic waste water treatment, environmental resource management and impact assessment, water quality management for decisionmakers, low-coast water supply and sanitation, polder development.

Renseignements : IHE, PO Box 3015, 2601 DA Delft, The Netherlands.

\section{Pôle européen d'enseignement à distance}

SCOLAQUA, gestion multicritère de l'eau - méthodes et stratégies. Formation niveau maîtrise de 50 heures.

Thèmes: principes de méthodologie multicritère et modélisation/eau, marché régional de l'eau, gestion intégrée d'un bassin versant (cours en anglais), aménagement multiobjectif de plans d'eau (cours en anglais), l'eau et les loisirs/questions sociologiques, l'usine d'épuration de la ville de Rennes.

Renseignements: CNED, 7, rue du Clos-Courtel. 35050 Rennes Cedex, Tél. : 99631188

\section{Centre de formation et de docu- mentation sur l'environnement industriel}

Stages, séminaires et stages «sur mesure». Parmi les stages :

- Les législations françaises et européennes concernant la pollution des eaux.

- Traitement des eaux usées et des boues industrielles.

- L'impact des rejets polluants dans le milieu aquatique.
- La mesure de la pollution des eaux.

- Les procédés d'épuration : principes et technologies.

- La pollution des eaux souterraines et des sous-sols.

Renseignements - CFDE, 99, bd Malesherbes, 75008 Paris, Tél. : 45622151 .

\section{Université libre de Bruxelles}

Cours sur la gestion de l'information en science et technologie (MIST 2) qui traite particulièrement de l'eau et de l'environnement, en anglais.

Renseignements : MIST 2, University Library. Free University Brussels, Pleinlaan 2. 1050 Brussels. Belgique.

\section{Ministère de l'environnement - Mission Défense/Office international de l'eau}

Programme de sensibilisation des maires et de leurs exploitants de réseaux d'eau potable aux problèmes de sécurité des installations. Programme de la journée de formation: étude de cas de pollutions accidentelles, dangers (sécheresse, pollution, contamination, interruption de la fourniture d'électricité). plans de secours, organisation de la sécurité en eau potable (dispositifs de protection et programme de sécurisation, détection et neutralisation des pollutions, solutions de secours).

Renseignements : Katia Bitner, Office international de I'eau, rue Edouard-Chamberland, 87065 Limoges Cedex, Tél. : 55114770 , Fax: 55777115 ou Michel Mesny, Ministère de l'environnement. 14, bd Général-Leclerc, 92524 Neuilly-sur-Seine Cedex, Tél. : 408185 95, Fax : 40818597.

\section{ENGREF}

L'Ecole nationale du génie rural, des eaux et des forêts propose des sessions de formation continue dans divers domaines dont : gestion de l'eau, mesures agri-environnementales, faune et flore, économie de l'environnement. outils de gestion pour l'entreprise, aménagement de carrières.

Renseignements: ENGREF, Formation continue, 19. av du Maine, 75732 Paris Cedex 15, Tél. 454989 12. Fax : 45498827.

\section{INP Formation continue, Toulouse}

Propose à des diplômés de niveau bac +2 (DUT, BTS, DEUG...) suivi de 3 années de réelle expérience professionnelle en qualité de technicien dans un domaine proche de la filière choisie de devenir ingénieurs par la voie de la formation continue. Cycle préparatoire de 16 à 18 mois par correspondance +2 ans d'études à temps plein. Parmi les spécialités, agronomie, chimie, génie chimique, hydraulique, informatique et mathématiques appliquées.

Renseignements: INP Formation Continue, 6, allée Emile-Monse, BP 4038, 31029 Toulouse Cedex, Tél. : 62242100, Fax : 62242101 . 